\title{
EVALUATION OF HISTORICAL HERITAGE DOCUMENTATION: REALITY BASED SURVEY AND DERIVATIVE MODELS
}

\author{
A. di Luggo ${ }^{* 1}$, M. Campi ${ }^{1}$, L. Repola ${ }^{2}$, V. Cera ${ }^{3}$, S. Scandurra ${ }^{1}$, M. Pulcrano ${ }^{1}$, M. Falcone ${ }^{1}$ \\ ${ }^{1}$ Dept. of Architecture, University Federico II of Naples, Italy - (m.campi, a.diluggo, simona.scandurra, \\ margherita.pulcrano)@unina.it; marikafalcone24@gmail.com \\ ${ }^{2}$ University Suor Orsola Benincasa, Naples, Italy - repolaleopoldo@ gmail.com \\ ${ }^{3}$ Urban/Eco Research Center, University Federico II, Naples, Italy - valeria.cera@ unina.it
}

\section{Commission II}

KEY WORDS: Data Integration, TLS, Time-of-Flight camera, Terrestrial Photogrammetry, 3D modeling, Derivative Models, Digital Documentation, Cultural Heritage.

\begin{abstract}
:
Three-dimensional acquisition systems for architecture have significantly evolved over just a few decades, with them allowing point clouds to be generated through active and passive optical sensor equipment.

Accuracy levels vary considerably in relation to both the equipment and techniques used, with the data obtained acting as a scaffolding for the creation of derived models that allow specific analyses to be carried out.

Ongoing research on Palazzo Donn'Anna, a Neapolitan sixteenth-century building of particular historical and artistic value, is being carried out in this context and the first results are presented in this paper.

The entire building has been the subject of an instrumental survey. The north-east façade was proposed as a case-study for the experimentation of diversified reality-based sensors so as to compare the accuracy and precision of the data. The comparison was also aimed at evaluating the performance of some processing softwares. Finally, in order to obtain an estimate of the data in the transcription from the point cloud to a derived 3D model, the reproduction of the same portion of the prospectus in a derivative model of both objectoriented and NURBS types was experimented.
\end{abstract}

\section{INTRODUCTION}

\subsection{Motivation and aim}

The widespread presence of historic buildings with an unusual configuration throughout the Neapolitan territory have led to the creation of a project of knowledge and dissemination of this heritage with an integrated vision of the knowledge necessary to study them. The existence of a building that has been inserted as a link between the land and sea along the coast of Naples and which reveals a complex history in the plasticity of its variable forms, provided the opportunity to apply the most well-known and contemporary surveying technologies and modelling processes to critically investigate the limits and potential that such systems show in complex and unusual operating scenarios. The study was therefore articulated with the aim of pursuing different objectives:

- 3D reconstruction of the whole building (outer skin, interior spaces, undergrounds and cavities);

- Combination of UAV and terrestrial image acquisition with terrestrial laser scans;

- Use and examination of the Riegl Vz400i scanner under practical and unusual conditions;

- Comparison of the results deriving from the different acquisition techniques;

- Use, comparison and integration of data from georadar and 3D muography for the analysis of the subsoil;

- use of data for restoration work and dissemination purposes.

The study was started in October 2018 and the paper presents some intermediate results of the project, with a general vision and the initial results being published by Repola et al. (2019).
An integrated approach to the study and collection of information related to historical heritage has long been a standard in the scientific community (Herráez et al., 2016; Hoon and Hong, 2019; Tucci et al., 2019). The collaboration between different universities has made it possible to carry out integrated technological and operational studies with a combination of expertise and know-how that is not always so easily achievable in order to know, celebrate and disseminate the culture of spaces and unusual architecture. [M.C.]

\subsection{Case Study: Palazzo Donn'Anna}

Palazzo Donn'Anna is one of the most significant examples of Neapolitan Baroque dating back to the 17th century (Belli, 2017). Built based on a project by Cosimo Fanzago, the work has never been fully completed. In particular, the north-east elevation upon which the study is based retains the charm of an ancient ruin, with windows alternating with openings without frames, the ruined and never plastered tuff wall, along with the semi-underground caves wetted by the sea. What makes the architectural work extremely characteristic is its particular position with respect to the sea since Palazzo Donn'Anna is built on a protrusion of the tuff bank in Posillipo, with it being connected to the coast only on one side, thus making the other three fronts unreachable. To document its dimensions and complex shapes, it was therefore necessary to use no-contact tools, modulating the choice of the specific instrument based on the characteristics of the surfaces surveyed and the morphology of the places, with the aim of creating a data-bank useful to record its current state, while also structuring the knowledge, valorisation and conservation processes. [S.S.]

\footnotetext{
Corresponding author
} 


\subsection{Applied methodology and theoretical issues}

The north-east elevation of the building has been proposed as an example of the procedures carried out for the comparison and integration of the data obtained from the two metric survey techniques, i.e. digital terrestrial photogrammetry and laser acquisition, in consideration of the advantages of both acquisition methodologies in the declination to a case study with a distinct singularity, in historical, artistic and architectural terms.

The research on the subject is varied (Liang et al., 2018; Adamopoulos et al., 2017; Yang et al., 2015; Remondino, 2011; Vosselman, 2002), and presents a variety of hardware and software solutions that can be used in homogeneity of the methodological approach.

For the study on Palazzo Donn'Anna, the laser acquisitions were carried out with a Riegl vz400i flight time instrument and the photographic acquisitions with a Canon EOS100D reflex camera. The data were first processed in applications dedicated to the specific type of acquisition (RiscanPro for the laser acquisitions and Agisoft Metashape for the photographic acquisitions) and then subsequently imported into the Reality Capture application to be integrated into a single metric and photorealistic result.

This procedure made it possible to highlight the differences of the image-based data in terms of the quantity and quality of the points processed by the different applications, along with the results from the comparison and integration with the laser scanner data.

Subsequently, the reproduction of the same portion of the elevation was studied in both a NURBS model as well as an object-oriented infographic model to evaluate the data in the transcription from the point cloud to a derived 3D model.

In the first case, the software Rhinoceros was used, along with the support of the polygonal mesh model deriving from the point cloud, which resulted in a model that strongly resembled the real artefact; in the second case, the software Autodesk Revit was chosen, importing and using the range-based point cloud as the scaffolding.

In recent years, the construction of object-oriented models to which to attach information that goes beyond the geometric and dimensional appearance is at the centre of the research of the scientific community (Lo Turco and Santagati, 2016; Bolognesi and Garagnani, 2018), from both the technological and methodological points of view, since it raises several questions related to the most suitable procedures and tools in the documentation of forms and meanings related to an architectural artefact. While on the one hand, image-based and range-based acquisition technologies provide effective visual data that are particularly accurate from a metric point of view, on the other, there is three-dimensional modelling that is characterized, as it is well-known, by a thick indistinct mass of points, for which the meaning - intrinsic and/or extrinsic - of what they represent is missing. Conversely, in returning these forms to threedimensional derivative models, or re-modelled according to procedures within the framework of Building Information Modelling, it is possible to give a univocal meaning to the individual parts making up the digital artefact, also associating rich information databases. Nevertheless, given the particular conformations that characterize historical architecture, the investigated artefact cannot always be replicated on BIM platforms, since the modelling tools they offer do not allow to obtain a graphic result of accuracy and verisimilitude comparable to reality-based surveys. The segmentation of the point clouds in order to create smart-objects corresponding to the real elements of the built architecture is a complex process, for which the recognition, coding and translation phase does not offer fully satisfactory solutions where automatisms are used (Russo and Guidi, 2011). As part of the study carried out on Palazzo
Donn'Anna, it was decided to use the BIM model as a master model, or rather as a place in which to bring together detailed information of a different nature, without necessarily replicating the forms of reality in an as-built inside the platform, where other connectable instruments (NURBS models and point clouds) provided a fully valid metric and geometric result. [S.S]

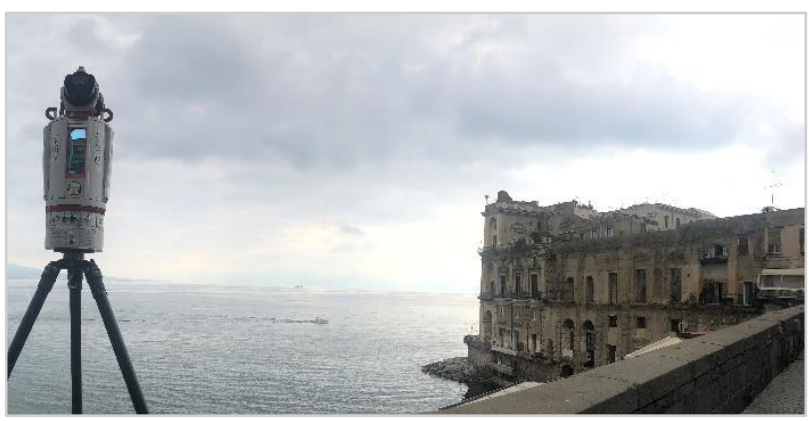

Figure 1. Scanning position on Via Posillipo

\section{FROM DATA ACQUISITION TO COMPARISON}

\subsection{TLS survey}

For the TLS survey, a Riegl vz400i time-of-flight scanner, equipped with a reflex camera, was used. The instrument was chosen for its long acquisition range $(0.5 \mathrm{~m}$ at $800 \mathrm{~m}$ with a resolution of $3 \mathrm{~mm}$ at $100 \mathrm{~m}$ ), since the presence of the sea and the beach led to the placing of scanning points particularly far away from the building.

In the placing of the scanning points useful for the threedimensional metric acquisition of Palazzo Donn'Anna, a path was defined according to a closed polygonal, in order to verify the average deviation of the clouds following the alignment procedures. The polygonal was designed so as to converge, through two acquisition paths, towards the sea terrace of the south-west elevation and towards the beach at the foot of the north-east elevation.

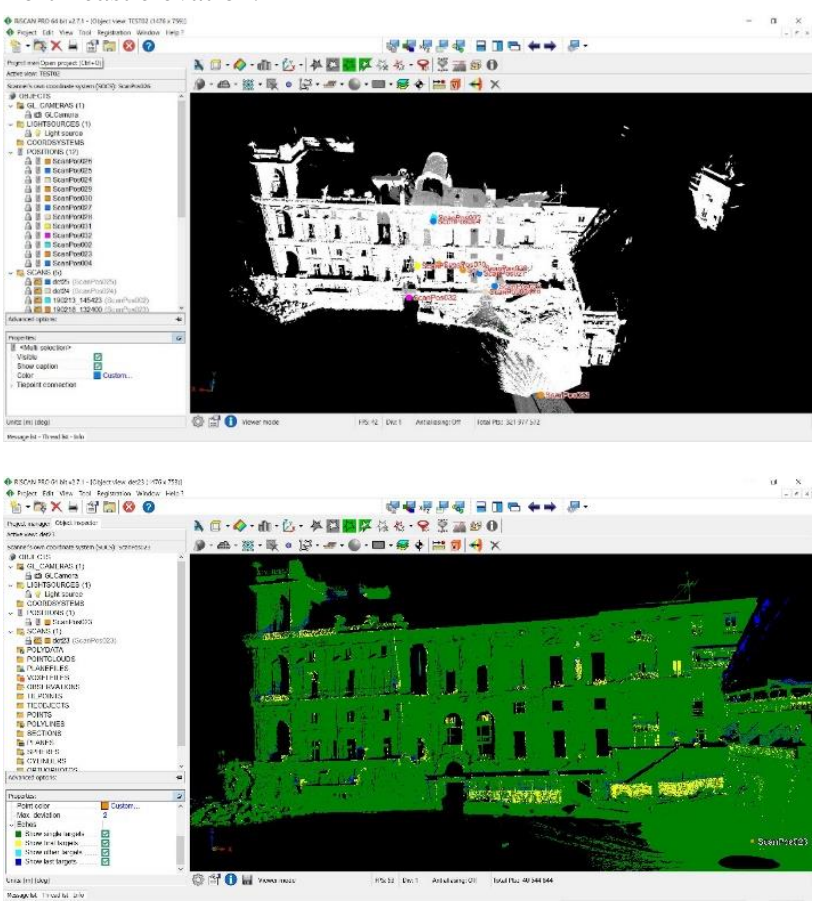

Figure 2. Point cloud in RiscanPro: color mode multiechos underscore targets 


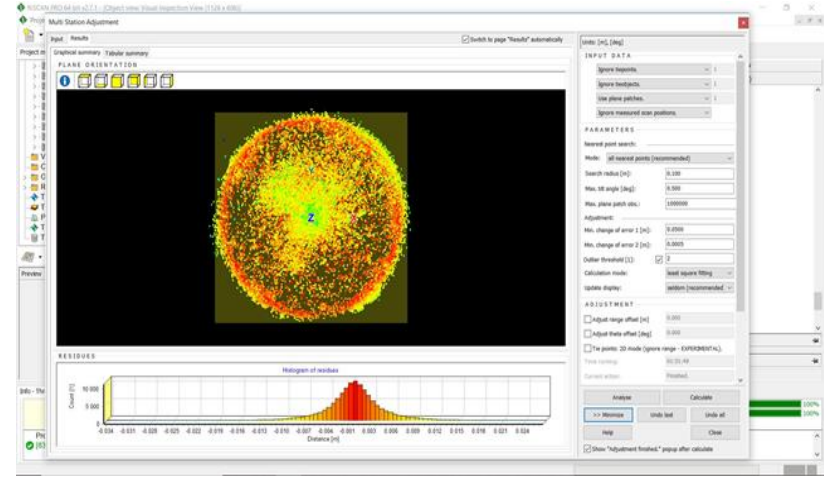

Figure 3. Detailed scan on flat circular reflector

Focusing on the north-east elevation as the subject of the present study, the acquisition took place from three scanning positions, one of which was located along Via Posillipo (Figure 1), about $12 \mathrm{~m}$ above sea level and $75 \mathrm{~m}$ from the nearest point of the elevation, two at the height of the beach, $50 \mathrm{~m}$ and $65 \mathrm{~m}$ respectively from the nearest point of the elevation. In view of the particular complexity of the elevation due to its poor conditions, the presence of different types of architectural elements and materials (in particular the presence of plastic nets to give protection from the falling of stone materials), as well as the unfinished volumes characterizing the elevation, the acquisitions were taken with different instrument settings.

It was chosen to set a fixed resolution of $3 \mathrm{~mm}$ for distances of $10 \mathrm{~m}$, while the laser frequency values varied between 300,600 and $1,200 \mathrm{kHz}$, so as to automatically identify and then move to different clusters, the different materials, while also selecting and eliminating the portions of the cloud related to weeds, plastic nets and metal reinforcement elements.

The alignment procedure (Figure 2; Figure 3) was verified progressively during the acquisition phases, having decided to manage the scanner and survey phases using a laptop directly connected to the instrument. This, in addition to allowing for the verification of the cloud quality - in terms of resolution and percentage of recovery area, avoiding excessive shaded areas allowed to re-calibrate the scanning project where the most distant areas or geometrically complex tufaceous cavities were not adequately documented. With the laptop interface of the RiscanPro software, it was possible to visualize and investigate in detail every single cloud acquired.

With the aim of greater accuracy, it was decided to place targets on the scene, of a three-dimensional and two-dimensional type, or 5 and $10 \mathrm{~cm}$ cylindrical reflectors as well as flat circular ones with a $5 \mathrm{~cm}$ diameter. Even if the instrument automatically identifies the targets present on the scene, in the acquisition phase it was decided to carry out, for each scan position, a scan at maximum resolution of only the targets, so that the alignment procedures were facilitated.

The workflow, if on the one hand required more on-site timing due to manual parameter management, on the other, made it possible to carry out fewer scan positions than those initially planned, since the constant verification of the results reduced redundancies and grey areas. Furthermore, the processing times of the data and the size in bytes of the models were reduced, making them easier to work in the management and modelling software.

The overall alignment of the point clouds was re-verified in the post processing phase using the standard RiscaPro software procedures, i.e. using the automatic registration and multi station adjustment functions. This procedure recorded alignment error levels of less than $2 \mathrm{~mm}$ for the areas closest to the instrument and about $6 \mathrm{~mm}$ for the furthest areas from the elevation.

Finally, the scans carried out were exported in the format .pts, preserving the totality of the acquired data. [L.R.]

\subsection{Image based acquisition}

To integrate the data acquired with the TLS, a photogrammetric survey was carried out to respond both to the need to match the accuracy of the metric data with a colorimetric representation capable of better expressing the quality of the building as well as to fill in some of the insufficient areas in the range-based data. In relation to the acquisition characteristics of the Riegl scanner and the modes, forced by the context with which the stations were carried out, the colorimetric data were not very refined where the elevation under study is recorded in a single frame.

At the same time, to maintain a high degree of accuracy and geometric precision in the photogrammetric model, an average GSD Ground Sample Distance of $4 \mathrm{~mm}$ was chosen.

Using a Canon EOS100D reflex, 141 total frames were acquired, at a land level corresponding to the height of the operator, with an average overlap along both capture lines ( $\mathrm{x}$ axis = overlap; $y$ axis $=$ sidelap) of about $60 \%$. In relation to the conformation of the facade and the limited possibilities of recovery dictated by the presence of the beach and the sea, it was decided to set the focal length to 55 and $24 \mathrm{~mm}$, integrating mixed shooting techniques, with parallel and convergent axes, thus recovering spatial information on different depth levels, while also limiting distortion errors.

No radiometric correction of the data in the pre-processing phase was necessary since the photographic bar was planned in advance, taking into account the variability of the lighting conditions, typical of external acquisitions and increased for the case study by the reflection of the water.

The images were oriented in two different Structure from motion applications, Agisoft Metashape and CapturingReality Reality Capture, in order to evaluate and compare the different performances and results obtained (Altuntas et al., 2016; Remondino et al., 2017).

Only the images with the highest quality parameters, based on the sharpness of the focus, were used for the orientation procedure of the photogrammetric datasets.

In line with the standard photogrammetric pipeline, the data set was oriented in Metashape according to the Self-Calibrating Bundle Adjustment. The search for correspondences, in this case, was based on a Future-Based Method since it did not use specific targets in the scene for photogrammetric acquisitions, believing that the list of corresponding pairs could be constructed by exploiting recognizable natural points and geometries as features. A scattered cloud of 76.225 points was then extracted. Subsequently, the homologous points, derived from the calculation of the internal and external orientation parameters, were used to produce a dense polygonal model using dense image matching algorithms. The result was a triangular mesh consisting of $8,473,113$ faces and 4,259,490 vertices.

The same data were processed simultaneously in the CapturingReality application. By subjecting the frames to an initial alignment process for the automatic computation of their position and orientation in the scene, the polygonal model was derived from the calculation of depth maps. In order to obtain an accurate but manageable three-dimensional model, considering the amount of data that the software was able to extract, a slightly under-sampled point cloud was triangulated. The density of the cloud was filtered establishing a minimum distance between two successive points equal to $0.002 \mathrm{~m}$, with it being necessary to discard any points with an intensity less than 0.03 since they were considered to be inaccurate. Starting from the streamlined data, 
the polygonal surface was generated, with its texturing be based on the "multi-band" calculation method and a reduction of the images before texturing equal to 2 and the intuitive filling of the parts not included from the overlap of the colorimetric data.

A first comparison between the photogrammetric outputs allowed to highlight the different number of tie points extracted from the two applications starting from the same input data. It is worth noting how even with parameters not set to the maximum processing quality, Reality Capture extracted and processed a cloud much denser than Metashape, with a ratio of about 1 to 9 (Table 1 and Table 2). [V.C.]

\section{Front North East Realitv Capture (141 imaqes)}

\begin{tabular}{|c|c|c|c|}
\hline & Tie points & mesh & texture \\
\hline n. points & 654025 & 16715885 & \\
\hline n. faces & & 33427650 & \\
\hline settings & $\begin{array}{l}\text { Max features per mpx: } 10 \\
000 \\
\text { Max features per image: } 80 \\
000 \\
\text { Max feature reprojection } \\
\text { error: } 2\end{array}$ & $\begin{array}{l}\text { Normal model } \\
\text { Detail decimation factor: } 1\end{array}$ & $\begin{array}{l}\text { Coloring method: Mutti-band } \\
\begin{array}{l}\text { Downscale image } \\
\text { texturing : }\end{array} \\
\text { Texururing } \\
\text { Visibility-based }\end{array}$ \\
\hline
\end{tabular}

Table 1. Photogrammetric data process in Reality Capture

\begin{tabular}{|l|c|c|l|}
\hline \multicolumn{4}{|c|}{ Front North East__ Metashape (141 images) } \\
\hline & \multicolumn{1}{|c|}{ Tie points } & \multicolumn{1}{|c|}{ mesh texture } \\
\hline n. points & 76225 & 4259490 & \\
\hline n. faces & & 8473113 & \\
\hline settings & $\begin{array}{l}\text { Quality: Low } \\
\text { Generic }\end{array}$ & $\begin{array}{l}\text { Quality: High } \\
\text { Surface type: arbitrary }\end{array}$ & $\begin{array}{l}\text { Mapping mode: orthophoto } \\
\text { Blending mode: mosaic } \\
\text { Texture size: 4096x1 }\end{array}$ \\
\hline
\end{tabular}

Table 2. Photogrammetric data process in Metashape

\begin{tabular}{|c|c|c|c|}
\hline \multicolumn{4}{|c|}{ Front North East__Reality Capture (2 scans Riegl) } \\
\hline & Tie points & mesh & texture \\
\hline n. points & 402320 & 6997654 & \\
\hline n. faces & & 13986374 & \\
\hline settings & $\begin{array}{l}\text { Max features per mpx: } 1000 \\
000 \\
\text { Max features per image: } 1 \\
000000 \\
\text { Max feature reprojection } \\
\text { error: } 1\end{array}$ & $\begin{array}{l}\text { High model } \\
\text { Detail decimation factor: } \\
0,1\end{array}$ & 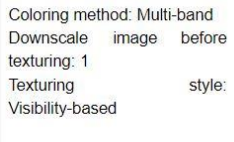 \\
\hline
\end{tabular}

Table 3. TLS data process in Reality Capture

\subsection{Data comparison}

In order to carry out an as complete as possible comparison between the different data, even the clouds acquired with laser scanners were processed in Reality Capture (Table 3 ).

The calculation parameters were set in order to compute all the scanning points, without the need for a subsampling due to the density of the starting data, which was considered adequate.
Starting from the extraction of tie points from the different scan clouds, exported in .ptx files with reflectance information, the triangular mesh was calculated by imposing the points captured with the laser scanner as polygon vertices. All the outputs obtained from the different acquisitions and processing were compared with reference to the clouds acquired with the Riegl laser scanner and recorded in RiscanPro.

The first comparison was carried out by aligning and calculating the deviation between the Riegl clouds and those processed in Reality Capture. The process was carried out in CloudCompare, subjecting the clouds to a rigid transformation based on the identification of at least 3 homologous points for each section. The result of the alignment, although iteratively improved thanks to the ICP algorithm implemented in the software, was not considered satisfactory. Therefore, starting from this first transformation, the two clouds were subjected to a Global Registration in Geomagic, this time obtaining an average deviation of $0.02 \mathrm{~m}$ compared to the $0.10 \mathrm{~m}$ previously registered. The punctual distance between the two models was recalculated in CloudCompare confirming the maximum deviation value recorded in Geomagic (Figure 4).

In the same way, all the models described above were compared two by two in CloudCompare with the same procedure (Georgopoulos, 2017; Luhmann et al., 2019) (Figure 5).

The dataset consisting of the Riegl scans was always used as a reference for the registration procedure with the ICP. Starting from the identification of homologous points - from a minimum number of 3 to a maximum of 6 - a rigid roto-translation was applied to each dataset to minimize the average distance between the two groups of data. The cloud/cloud distance values, calculated each time, showed a maximum adherence between them near the station points and an increase in the deviation, always contained in $2 \mathrm{~cm}$ on average, upon moving away from the point acquisition, as was easily predictable in relation to the aberrations of the optics. [V.C.]
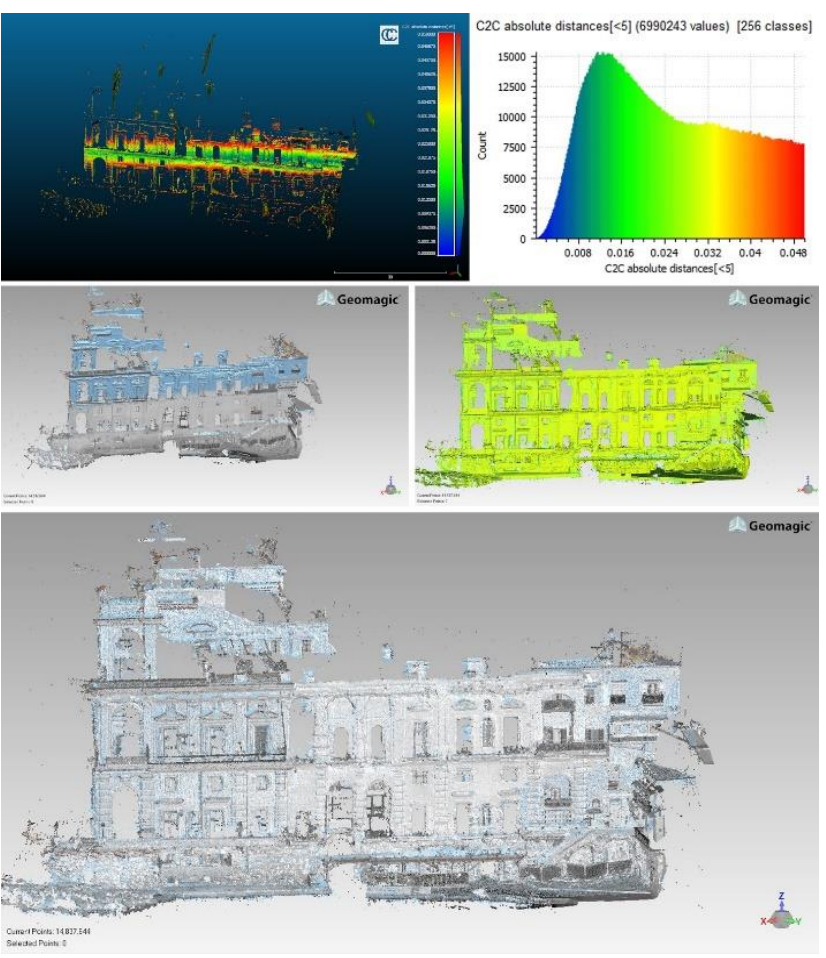

Figure 4. Alignment and deviation computing between the TLS clouds, in CloudCompare (above) and Geomagic (below) 

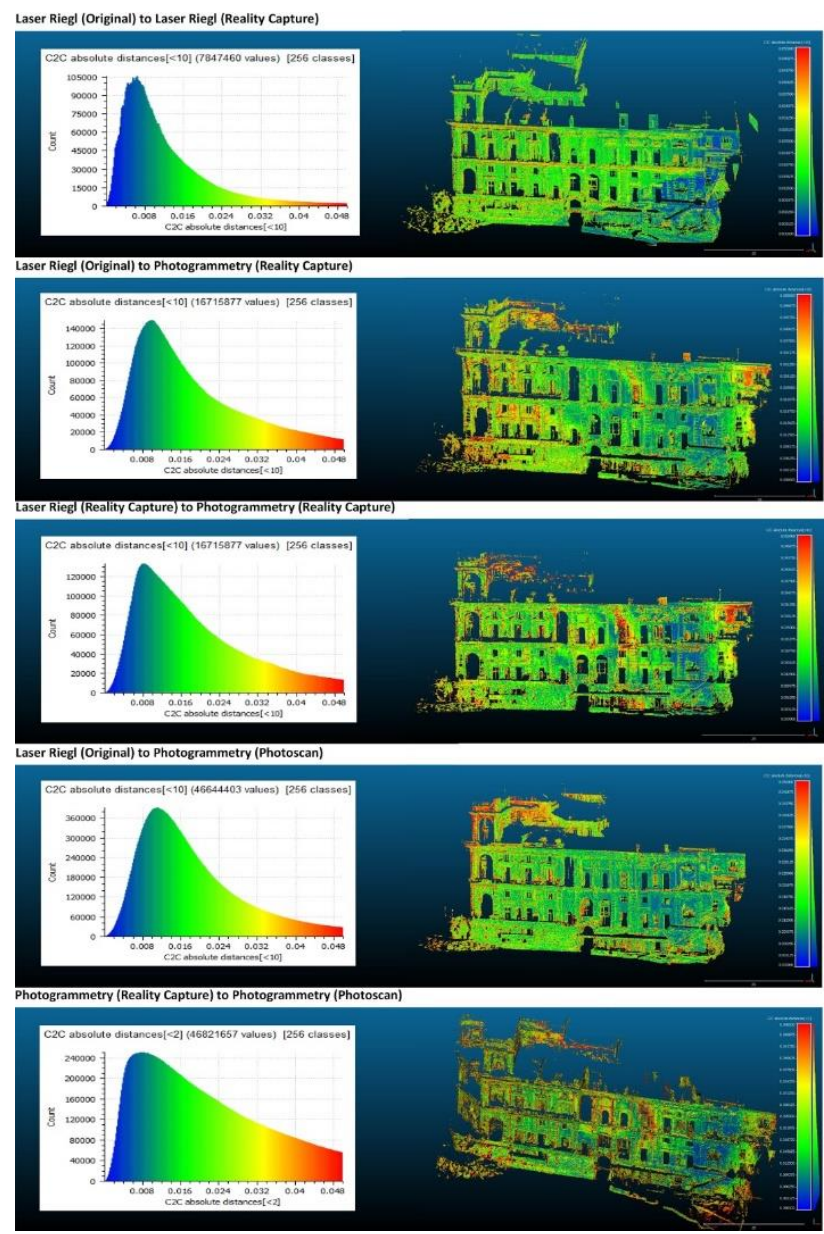

Figure 5. Deviation maps of the different outputs, compared in pairs

\section{DERIVATED MODEL}

\subsection{NURBS modeling}

The digital result returned by the Riegl vz400i laser scanner was the initial data upon which to intervene with post processing operations through which the point model was transformed into a polygonal model (mesh, NURBS).

The three-dimensional digitalization process (Giordano and Repola, 2016) began with reference to the north-east elevation, which, in this specific case, turned out to be inverse with respect to the canonical process in which the two-dimensional drawings (plan, section and elevation) are the starting point.

Three-dimensional modelling was only carried out on the southern portion of the north-east elevation, imported as a polygonal model, with medium resolution parameters, directly in the 3D CAD environment, and specifically in the Rhinoceros software, exploiting the use of NURBS modelling (Non-Uniform Rational B-Spline).

The result of the mesh model in this case has the largest number of polygons on the north side of the elevation, while in the angular part (on all three levels) it shows a considerable reduction of the polygons due to the limited possibility of shooting point clouds during the on-site acquisition phases (Bevilacqua et al., 2017).

Starting from this polygonal model through a series of progressive sections, obtained with parallel and orthogonal planes, the three-dimensional modelling was carried out, resulting within the virtual environment in the model with the example geometries of the object under study.

It is worth taking into account how the mesh model has a rather coarse appearance since it is obtained by the simple union of points in the space with a mesh that, however dense, always leaves gaps between one point and another. Whereas, wanting to obtain a more accurate result, very close to reality, it was necessary to export single portions of the polygonal model with parameters at maximum resolution from the CapturingReality Reality Capture application. The individual exported parts had a very high infill of the polygonal mesh with a greater detail of the architectural elements (Figure 6). In this way, proceeding with the same modelling technique, it was possible to obtain two three-dimensional models in which it was possible to compare and evaluate the accuracy of the data. [M.F.]
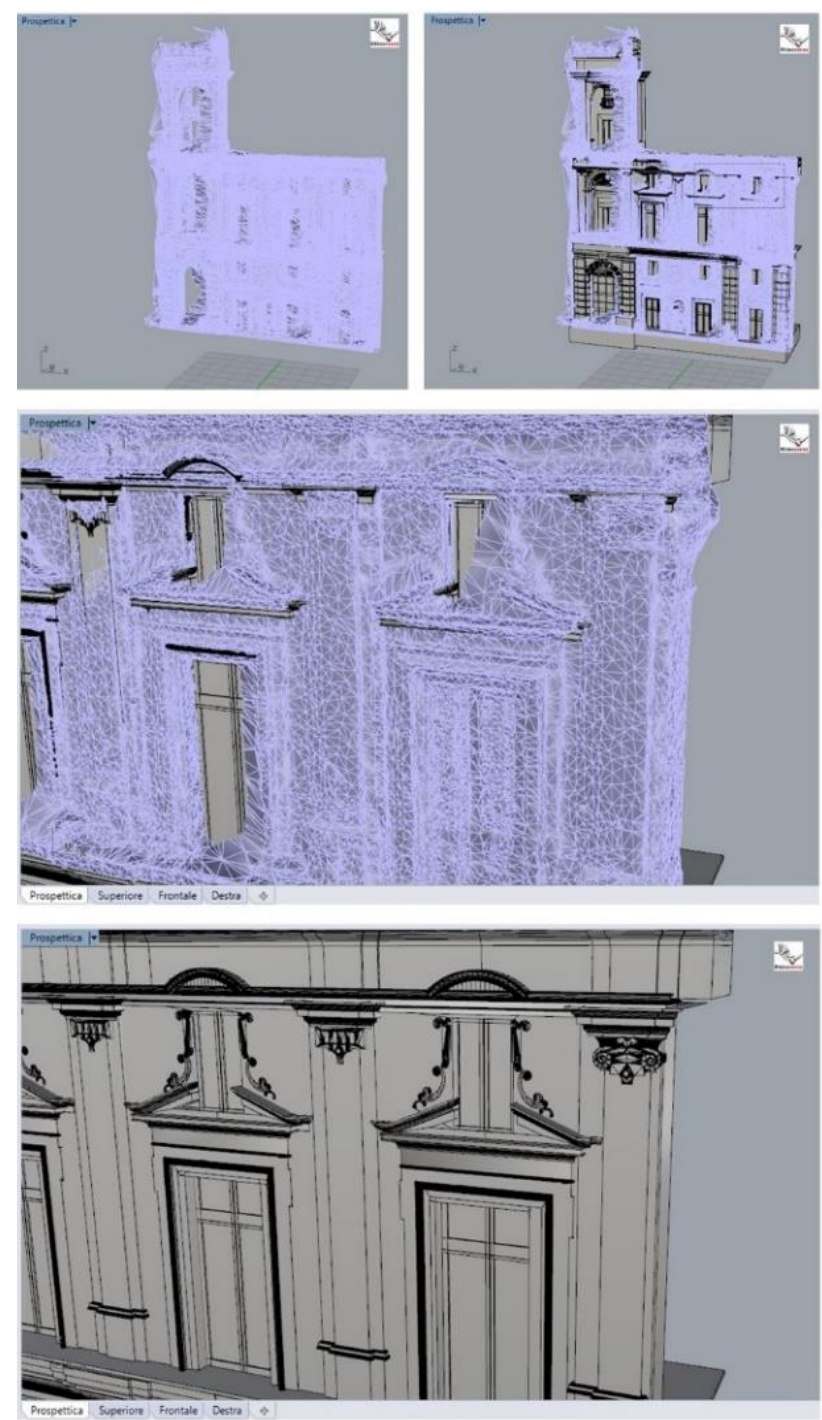

Figure 6. Polygonal model (mesh) and NURBS model in adherence to the mesh, in Rhinoceros (above); Detail of the decorative elements in NURBS model (below)

\subsection{Object Model}

For the graphic restitution of the acquired data, it was decided to use the BIM methodology and the Autodesk platform to translate 
and manage the transition from the point model to the informative and representative, model of Palazzo Donn'Anna.

In order to construct the HBIM model of Palazzo Donn'Anna, it was necessary to reflect on the theoretical and methodological implications underlying the application of BIM to historical heritage since, along with the undoubted advantages and potential of the method (Bolognesi and Garagnani, 2018), the particular conformation of the building and at the same time, the absence of instruments dedicated to the historical heritage in the current BIM platforms, makes it difficult to pigeonhole the elements that make up Palazzo Donn'Anna into pre-defined and standardized structures. Moreover, while adapting the instruments to obtain a geometrically coherent result with the reality-based survey data, the times and measures in the geometric and semantic characterization of the models do not always satisfy the accuracy requirements required by a BIM model which, by its very nature, tends to be the total simulation of reality. Data structuring methodologies are currently being tested, which can respond simultaneously to the metric, geometric and syntactic requirements of an architecture, in order to build a single database as a synthesis of numerous heterogeneous pieces of information.

For the modelling of the architectural and decorative elements of Palazzo Donn'Anna, it was therefore necessary to first break it down into constitutive elements, so as to identify the family that best represented its form and meaning. Thus, walls, floors, windows, etc. were modelled using the corresponding families system, which were loadable following the logic of interrelation of the real element with the virtual one according to two-way relationships.

For other elements of the historical architecture not foreseen by the platform used, this logic was forced by constructing objects that, if by form, meet the pre-established requirements for representation and are adapted to the tools offered by meaning. The pilasters that characterize the architecture of the building are an example, marking the compositional rhythm of the facades, and which, as it is well-known, are elements not present in the Revit software. It was decided to resort to loadable families and shape the form in the template "generic model based on wall" so as to maintain, at least, the relationality of the object with the host element, i.e. the wall.

From a purely geometric point of view, each element was constructed by applying the simplification criteria of the forms, depending on the level of detail identified, with a medium-low level being considered sufficient for the specific case study of Palazzo Donn'Anna. In this way, an overall homogeneous model was obtained and, at the same time, the modelling times and operations were contained. Furthermore, to make the information comprehensive, where possible, additional data were associated through external links (Monaco, et al., 2019) (Figure 7). The capitals at the end of the façade pilasters are an example, which, conceived as simple volumes, were equipped with the corresponding portions of both the point cloud and the NURBS model.

In the discretization of the forms, the individual smart-objects (Biagini and Donato, 2014; Chiabrando, et al., 2017) were broken down into sub-elements, subsequently related by means of nested families for a correct parameterization of the overall element. The window with the tympanum was subdivided into a tympanum, a frame, a lower frame, an upper frame and external blinds, all of which, in turn, were broken down into simpler elements and related through parameters (Figure 8).

Even for the different sub-elements, the template considered the most appropriate for the modelling objectives was chosen from time to time and, when allowed, it was later characterized as a "window" in order to make all the parts coherent with the final family. In addition to the geometric parameters, which allowed

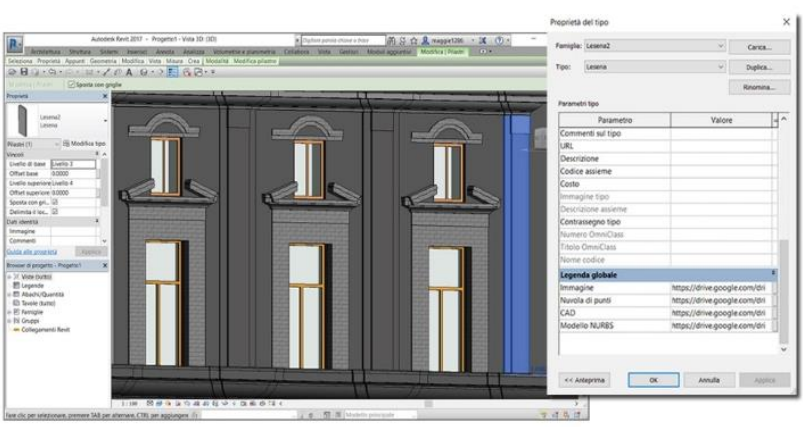

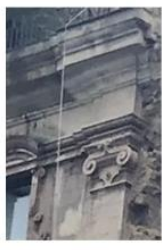

Photo

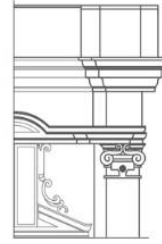

CAD

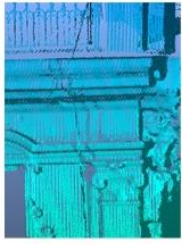

Point Cloud

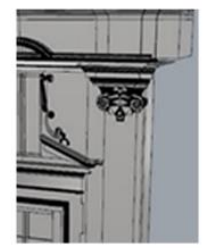

NURBS Model
Figure 7. Every type of information has been linked to HBIM model.

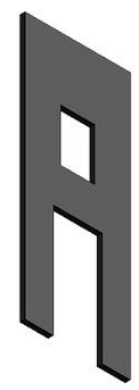

LAW
level of detail

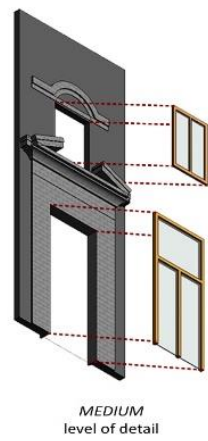

$\underset{\text { MEDIUM }}{\text { level of detail }}$

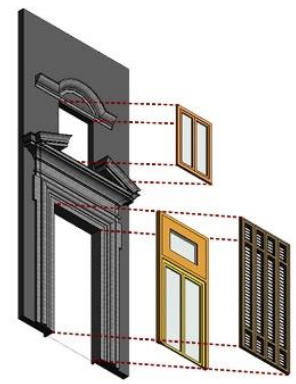

HIGH
level of detail
Figure 8 . The window with the tympanum has been modelled with different level of detail evaluating different possible uses.

to manage the dimensions of the shape, each smart-object was characterized with parameters that defined its material and descriptive attributes.

Finally, particular attention was paid to the methods of representation according to the different levels of graphicization (high, medium, low) envisaged by the Revit software, linking the choices made not so much to the information content - variable with respect to the various components of the model - but rather to the graphic synthesis of the element. Specifically, after setting codification rules in relation to traditional forms of representation, the sub-elements of the smart-objects were modelled and characterized - with the types of lines and thicknesses - to adequately respond to the level of detail set for the BIM model. At the low level, for example, there is a simplification of the forms that allows for the identification of the element and the reading of its general dimensional characteristics. As the graphic level increases, so does the graphic detail, until the display at the maximum level corresponds to reality, making it possible to read the different parts that compose it. [M.P.] 


\section{CONCLUSIONS}

The wide variety of techniques and tools available today make the digitization of Historical-Architectural Heritage a field of research that is always open and in continuous critical evolution. The study presented in this contribution is part of the current debate of investigation, focusing on two issues in particular: the acquisition of instrumental survey data and the construction of derivative models. The specificities of the analysed product, along with the characteristics of the hardware and software tools used have led to the formulation of specific considerations on the workflow.

First of all, the comparison of the results obtained from the different three-dimensional acquisitions made it possible to evaluate and complete the metric acquisitions through comparisons and additions.

Subsequently, the goal of conforming a database with different levels of detail led to considering and organizing the BIM model as an informative support, simplified from a graphic-visual point of view, but capable of associating geo-referenced detail data on the model and structured according to different ways of representation: point cloud, NURBS model, three-dimensional images, geometric studies, historical insights, etc., necessary for understanding the real element in all its features and peculiarities. This operational solution highlights how the translation of the point cloud into infographics of historical architecture is still a complex process, influenced by multiple factors linked, in particular, to the forms of construction of the historical buildings as well as to their relative topological and semantic characterization. [A.d.L.]

\section{REFERENCES}

Adamopoulos, E., Tsilimantou, E., Keramidas, V., Apostolopoulou, M., Karoglou, M., Tapinaki, S., Ioannidis, C., Georgopoulos, A., Moropoulou, A., 2017. Multi-sensor documentation of metric and qualitative information of historic stone structures. Int. Arch. Photogramm. Remote Sens. Spatial Inf. Sci, IV-2/W2, 1-8.

Altuntas, C., Yildiz, F., and Scaioni, M., 2016. Laser Scanning and Data Integration for Three-Dimensional Digital Recording of Complex Historical Structures: The Case of Mevlana Museum. ISPRS Int. J. Geo-Inf. 201, 5, 18.

Belli, P. (a cura di), 2017. Palazzo Donn'Anna. Storia, arte e natura, Torino, Umberto Allemandi, ISBN: 88-422-2425-1.

Bevilacqua, V., Trotta, G., and Brunetti, A., 2017. Photogrammetric mesh and 3D points cloud recostruction: a generic algorithm optimization procedure. Communications in Computer and Information Science, 708, Springer, Cham: 65-76.

Bolognesi, C., and Garagnani, S., 2018. From a point cloud survey to a mass 3D modelling: Renaissance HBIM in Poggio a Caiano. Int. Arch. Photogramm. Remote Sens. Spatial Inf. Sci., XLII-2: 117-123.

Biagini, C., and Donato, V., 2014. Building Object Models (BOMS) for the documentation of Historical Building Heritage. $V$ Congreso Internacional de Expresion Grafica.

Chiabrando, F., Lo Turco, M., and Santagati, C., 2017. Digital Invasion: from Point Clouds to Historical Building Object Modeling (H-BOM) of a Unesco WHL Site. Int. Arch.
Photogramm. Remote Sens. Spatial Inf. Sci., XLII-2/W3: 171178.

Georgopoulos, A., 2017. Data Acquisition for the Geometric Documentation of Cultural Heritage. Ioannides M., MagnenatThalmann N., Papagiannakis G. (eds) Mixed Reality and Gamification for Cultural Heritage, Springer, Cham: 29-73.

Giordano, A., Repola, L., 2016. The double dynamics of knowledge: reality and virtual reality in the enhancement project of Torre Maggiore of Villa Rufolo, Ravello, DisegnareCon, vol. 9/n.17, 12.1/12.9.

Herráez, J.; Martínez, J.C.; Coll, E.; Martín, M.T.; Rodríguez, J., 2016. 3D modeling by means of videogrammetry and laser scanners for reverse engineering. Measurement, 87, 216-227.

Hoon Jo, Y. and Hong, S., 2019. Three-Dimensional Digital Documentation of Cultural Heritage Site Based on the Convergence of Terrestrial Laser Scanning and Unmanned Aerial Vehicle Photogrammetry. ISPRS Int. J. Geo-Inf. 2019, 8, 53.

Liang, H.; Li, W.; Lai, S.; Zhu, L.; Jiang, W.; Zhang, Q., 2018. The integration of terrestrial laser scanning and terrestrial and unmanned aerial vehicle digital photogrammetry for the documentation of Chinese classical gardens - A case study of Huanxiu Shanzhuang, Suzhou, China. J. Cult. Herit., 33, 222230.

Lo Turco, M., and Santagati, C., 2016. From structure from motion to historical building information modeling: populating a semantic aware library of architectural elements. Journal of Electronic Imaging, 26(1): 1-12.

Luhmann, T., Chizhova, M., Gorkovchuk, D., Hastedt, H., Chachava, N., and Lekveishvili, N., 2019. Combination of terrestrial laserscanning, uav and close-range photogrammetry for $3 \mathrm{~d}$ reconstruction of complex churches in georgia. Int. Arch. Photogramm. Remote Sens. Spatial Inf. Sci., XLII-2/W11: 753761.

Monaco, S., Siconolfi, M., and di Luggo, A., 2019. ExistingBIM: integrated survey procedures for the management of modern architecture, Int. Arch. Photogramm. Remote Sens. Spatial Inf. Sci., XLII-2/W9, 495-500, https://doi.org/10.5194/isprs-archives-XLII-2-W9-495-2019.

Remondino, F., 2011. Heritage Recording and 3D Modeling with Photogrammetry and 3D Scanning. Remote Sensing, 3(6), 11041138 .

Remondino, F., Nocerino, E., Menna, F., and Toschi, I., 2017. A critical review of automated photogrammetry processing of large dataset. Int. Arch. Photogramm. Remote Sens. Spatial Inf. Sci., XLII-2/W5: 591-599.

Repola, L., di Luggo, A., Campi, M., Cera, V., Scandurra, S., Pulcrano, M., Scotto di Carlo, N., Pisano, F., 2019. La rappresentazione quale specchio del reale: le nuove tecnologie a servizio della conoscenza per la documentazione di ambienti complessi. Paolo Belardi (a cura di), Riflessioni. L'arte del disegno/il disegno dell'arte, Gangemi editore, 961-968. 
Russo, M., and Guidi, G., 2011. La modellazione digitale realitybased ed interpretativa per la valorizzazione del patrimonio culturale. La Ricerca nel Disegno di Design, Maggioli Editore: 207.

Tucci, G., Rihal. S., Betti, M., Conti, A., Fiorini, L., Kovacevic, V.C., Bartoli, G., 2019. Ground based 3D modelling (photogrammetry and TLS) - survey, documentation and structural assessment of XX century cultural heritage in India A case study of the masonry vaults in Dehradun. The International Archives of the Photogrammetry, Remote Sensing and Spatial Information Sciences, XLII-2/W11, 2019, 11051111 .

Vosselman, R., 2002. Fusion of Laser Scanning Data, Maps, and Aerial Photographs for Building, Proceedings of the IEEE International Geoscience and Remote Sensing Symposium, Toronto, ON, Canada, 24-28 June 2002, 85-88.

Yang, B.; Zang, Y.; Dong, Z.; Huang, R., 2015. An automated method to register airborne and terrestrial laser scanning. ISPRS J. Photogramm., 109, 62-76. 